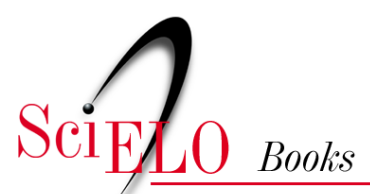

\title{
7. Comissões e grupos temáticos
}

\author{
Everardo Duarte Nunes
}

NUNES, E. D. Comissões e grupos temáticos. In: LIMA, N. T., and SANTANA, J. P., ed s. Saúde coletiva como compromisso: a trajetória da Abrasco [online]. Rio de Janeiro: Editora FIOCRUZ; Abrasco, 2006, pp. 187-214. ISBN: 978-65-5708-156-3. https://doi.org/10.7476/9786557081563.0008.

All the contents of this work, except where otherwise noted, is licensed under a Creative Commons Attribution 4.0 $\underline{\text { International license. }}$

Todo o conteúdo deste trabalho, exceto quando houver ressalva, é publicado sob a licença Creative Commons Atribição 4.0.

Todo el contenido de esta obra, excepto donde se indique lo contrario, está bajo licencia de la licencia $\underline{\text { Creative }}$ Commons Reconocimento 4.0. 


\section{Comissões e Grupos Temáticos}

Everardo Duarte Nunes

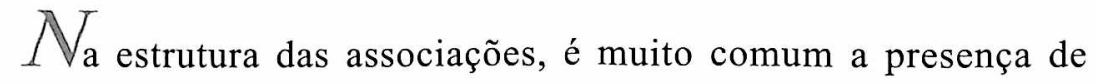
alguns órgãos internos que possibilitem o seu funcionamento e que muitas vezes são essenciais para a sua existência e desenvolvimento, especialmente no caso de associações com finalidades que se ampliam ao longo do tempo. Além disso, os interesses coletivos a serem partilhados necessitam de formas organizacionais que permitam agregar partes de um todo, estruturalmente consistentes e funcionalmente eficientes. Transferindo para as associações os pressupostos modernos das teorias organizacionais (Kauffman, 1993), podemos dizer que seus principais elementos são as pessoas, a estrutura, a tecnologia e o entorno em que funcionam. Reafirmamos que a estrutura consolida-se por meio do consenso dos interesses dos grupos constituintes. Também podemos dizer que as associações são estruturadas e estruturantes: constituem um campo instituído, mas possibilitam o aparecimento de novos atores coletivos. Em muitos casos, temas relevantes constituem o ponto de partida da formação de grupos e associações. Em verdade, não foi estranha à ciência, desde as suas origens, a constituição de associações congregando seus participantes e seus interesses especiais.

Nem sempre, estatutariamente, são definidas todas as estruturas internas das sociedades e associações; elas aparecem na medida em que se tornam imprescindíveis para o seu funcionamento. Este é o caso da Associação Brasileira de Pós-Graduação em Saúde Coletiva (Abrasco). Embora não constem de seu estatuto as instâncias denominadas comissões e grupos temáticos, elas estiveram presentes desde o início da instituição e foram se diversificando ao longo do tempo. 
Assim, torna-se importante que se dedique algum espaço aqui à análise das comissões e dos grupos temáticos. Ela terá como base as informações anteriormente compiladas em trabalhos (Belisário, 2002) e relatórios e aquelas fornecidas pelos coordenadores.

Atualmente, a Abrasco é composta por quatro comissões: Comissão de Ciências Sociais e Humanas em Saúde, Comissão de Ciência e Tecnologia, Comissão de Epidemiologia e Comissão de Políticas de Saúde, Planejamento e Gestão, além de 12 grupos temáticos: Acreditação Pedagógica, Comunicação e Saúde, Educação Popular e Saúde, Gênero e Saúde, Promoção da Saúde, Saúde do Trabalhador, Saúde e Ambiente, Saúde dos Povos Indígenas, Informação em Saúde, Profissões e Recursos Humanos, Saúde Mental, Vigilância Sanitária. ${ }^{1}$ A própria nomenclatura dessas comissões e grupos estabelece o amplo escopo de atividades a que se dedica a associação e, provavelmente, não completa a sua tarefa. Certamente alguns GTs poderão vir a se transformar em comissões.

A fim de situar as questões mais relevantes que têm sido objeto das comissões e dos grupos temáticos, apresentaremos uma análise separada de cada item.

\section{As CoMissões}

Utilizamos dois momentos assinalados por Belisário (2002:151), quando transcreve documentos que mostram a criação das comissões. No primeiro, que data de 1983, podemos ler:

as comissões têm função de contribuir para o estabelecimento das políticas de ensino e pesquisa da Associação nas respectivas áreas e desenvolver a programação editorial correspondente (...). Já as comissões executivas são estruturas funcionais temporárias, adjuntas à secretaria executiva, cuja finalidade é programar, executar e avaliar um projeto de objetivos definidos num tempo determinado.

Dez anos depois, em 1994, o documento da Abrasco assinala: "as comissões dentro de suas competências constituem-se como grupo assessor de processo de Saúde Coletiva e como suporte para as reflexões e tomada de decisões da diretoria". De acordo com os delineamentos iniciais, percebemos que essa forma de organização constitui uma "estratégia administrativa que permite a programação, execução e avaliação de uma grande quantidade de atividades de amplo alcance, com uma estrutura leve,

${ }^{1}$ Deixam de ser analisados alguns grupos temáticos em virtude de não dispormos de informações recentes. 
barata e funcional, que possui, além disso, amplo respaldo dos interessados" (Belisário, 2002:152).

\section{Comissão de Ciências Sociais e Humanas em Saúde}

Embora a Comissão de Ciências Sociais e Humanas em Saúde seja uma das mais antigas da Abrasco, originalmente designada como Comissão de Ciências Sociais, somente na década de 90 recebeu maior impulso. A realização do I Encontro de Ciências Sociais em Saúde, em setembro de 1993, em Belo Horizonte (MG), seguida da Oficina de Ciências Sociais em Saúde, realizada no Rio de Janeiro em abril de 1995; a publicação de uma coletânea dos textos (Canesqui, 1995) apresentados nessa reunião e a realização do I Congresso Brasileiro de Ciências Sociais em Saúde, em novembro de 1995, em Curitiba (PR), abriram as possibilidades da elaboração do I Plano Diretor da área, em 1977. Antes foram realizadas duas oficinas de trabalho: uma em Campinas (SP), em 1996, e outra por ocasião do V Congresso Brasileiro de Saúde Coletiva, em Águas de Lindóia (SP), em 1997.

O I Plano, além de retomar as iniciativas anteriores, elaborou um diagnóstico da situação e os principais problemas relacionados às questões de ensino nos diferentes níveis, à pesquisa e à prestação de serviços.

Em dezembro de 1999, foi realizado em São Paulo o II Congresso Brasileiro de Ciências Sociais em Saúde, que contou com o expressivo número de 578 inscritos. Desse total, 367 atenderam às informações solicitadas pela organização do evento, por meio das quais verificou-se que $33,8 \%$ deles procediam das ciências biológicas e biomédicas; 34,1\%, das ciências sociais e humanas; $1,1 \%$, das ciências da computação e matemáticas; $0,6 \%$, da comunicação e publicidade; $24 \%$ identificaram-se como docentes e pesquisadores e $5,7 \%$ como estudantes. Durante o congresso, foram realizados sete cursos com temática bastante variada; três conferências, ${ }^{2}$ duas palestras complementares ${ }^{3}$ e nove mesas-redondas, 54 comunicações coordenadas e 102 pôsteres. Observou-se no congresso a presença de participantes de todos os estados do país, com predomínio da Região Sudeste $(69,05 \%)$. Ressalte-se a qualidade dos trabalhos apresentados, que viriam a fazer parte da coletânea organizada por Paulete Goldenberg, Regina Maria Giffoni Marsiglia e Mara Helena de Andréa Gomes (Goldenberg, Marsiglia \& Gomes, 2003), conforme o Relatório do II Congresso Brasileiro de Ciências Sociais em Saúde, de 1999.

\footnotetext{
${ }^{2}$ Conferências proferidas por Gabriel Cohn, Everardo Duarte Nunes e Francisco de Oliveira.

${ }^{3}$ Palestras proferidas por Roberto Briceño de Leon.
} 
A realização do III Congresso de Ciências Sociais e Humanas em Saúde, realizado em Florianópolis (SC), evidenciou que a área está consolidada, contando com uma ampla e diversificada produção científica. Nesse congresso, foram inscritos 2.032 trabalhos, dos quais 443 apresentados oralmente e 1.589 sob a forma de pôsteres, e realizaram-se dez oficinas temáticas pré-congresso. O congresso contou com 1.800 participantes. Dentre os pontos analisados no Documento da Oficina do III Congresso de Ciências Sociais e Humanas em Saúde (2005), destacamos:

Um primeiro ponto a considerar é o da identificação e fortalecimento de veículos de difusão, de modo a ao mesmo tempo dar mais visibilidade e qualificação à produção da área. Propomos a identificação dos periódicos nacionais que concentram a maior parte da produção do campo, para que se proceda, se for o caso, ao upgrade de seu Qualis na Capes, bem como a proposição da chancela da Abrasco a editoras e/ou coleções que acolham a nossa produção.

Um segundo ponto é garantir a qualificação adequada da área de Ciências Sociais e Humanas em Saúde nos programas de Saúde Coletiva. Não é aceitável a ocorrência, como por vezes tem-se dado, da criação de novos programas ou áreas de concentração sem sequer a presença de um pesquisador qualificado na área.

Propomos ainda o fortalecimento da representação da sub-área [sic] nos diversos comitês de avaliação, como parte integrante que somos da área de Saúde Coletiva, e com uma presença à altura de nossa participação na área. Acreditamos ser necessário ainda reativar e melhorar o sistema de comunicação entre o Fórum de Coordenadores de Pós-Graduação da ABRASCO e os professores e pesquisadores das Ciências Sociais e Humanas em Saúde.

Citamos ainda, desse documento, os trechos seguintes:

Do ponto de vista estratégico, devemos buscar a construção de alianças com outras subáreas, Grupos de Trabalho e Comissões da Abrasco, sem prejuízo de se buscar apoios também nas Medicinas, Fisioterapia, Odontologia, Enfermagem, Farmácia e Nutrição, nossos parceiros nas áreas temáticas do Cnpq [sic] e da Capes. Ainda neste sentido, consideramos ser importante a interlocução com as próprias áreas de Ciências Sociais e Humanas que também sofrem com os excessos da normatização avaliativa.

Ainda neste sentido, entendemos que cabe às Ciências Sociais e Humanas a reflexão crítica sobre as concepções sobre 'ciência' e 'produção' acriticamente adotadas em nosso meio. A adoção de uma lógica de mercado, de competição excludente, que concentra no topo de pirâmides imaginárias os recursos não é nem justa, nem produtiva. É fundamental incentivar também 
a cooperação e solidariedade entre pesquisadores, buscando incentivar a criatividade científica e o livre pensamento.

No final do documento, as recomendações feitas visaram fortalecer as relações entre as comissões, entre instituições de fomento e de avaliação, situando de forma clara a especificidade da área, sem perda dos critérios de cientificidade e eticidade.

\section{Comissão de Ciência e Tecnologia}

A base dessas considerações relacionadas à Comissão de Ciência e Tecnologia é o documento elaborado pela comissão intitulado "Política Nacional de Ciência, Tecnologia e Inovação em Saúde: uma proposta". ${ }^{4}$ Os principais pontos indicam que uma política de C\&T em saúde deve estar

voltada para as necessidades de saúde da população e deve ter como objetivo principal desenvolver e otimizar os processos de absorção de conhecimento científico e tecnológico pelas indústrias, pelos serviços de saúde e pela sociedade. Isto significa analisar o esforço nacional de C\&T em saúde como um componente setorial do sistema de inovação brasileiro. No entanto, para compreender a pesquisa em saúde como um componente setorial do Sistema Nacional de Inovação, e remeter o objetivo geral da pesquisa em saúde às necessidades de saúde da população não se deve sugerir uma visão reducionista ou utilitarista da mesma. Pelo contrário, deve-se reconhecer a complexidade dos processos de produção de conhecimento científico e tecnológico.

Essas idéias são orientadoras de uma posição que enfatiza

a importância da pesquisa estratégica, no âmbito de uma agenda que incorpore potencialmente todo o leque da pesquisa científica e tecnológica que tenha como finalidade, imediata ou mediata, contribuir para a melhoria do estado de saúde da população e a busca da redução da desigualdade social no cuidado à saúde.

Ao salientar a presença de um 'patrimônio institucional', o documento aponta a existência de empecilhos para um aproveitamento integral de suas capacidades, que são a falta de coordenação e a baixa capacidade de articulação.

Ao reproduzirmos a proposta política da Comissão, verificamos que ela está atenta aos principais pontos que podem dimensionar a sua

\footnotetext{
${ }^{4}$ Resumo elaborado por Ana Cecília Faveret. Fonte: Sumário Executivo do documento "Política Nacional de Ciência, Tecnologia e Inovação em Saúde: uma proposta", da Comissão de C\&T da Abrasco. Disponível em: <http://www.abrasco.org.br/Boletins/bol84/bol84partic1. htm\#Ciência,\%20Tecnologia\%20e\%20Inovação\%20em\%20Saúde>. Acesso em: 21.abr.2006.
} 
efetiva presença na Saúde Coletiva. Essa proposta sustenta-se em cinco diretrizes gerais:

1. Busca da redução da desigualdade;

2. Construção de padrões éticos na prática da pesquisa;

3. Extensividade no que se refere à cadeia do conhecimento, bem como a 'inclusividade' no que toca aos atores (pesquisadores e demais recursos humanos);

4. Necessidade de sustentar a pesquisa em saúde como um exercício de lógicas complementares;

5. Necessidade de aumentar a capacidade indutora do sistema de fomento científico e tecnológico.

A Comissão não se esqueceu de apontar as estratégias necessárias à elaboração de uma agenda de pesquisa em saúde, orientada pelas diretrizes gerais da política de saúde. Também constam do documento aspectos ligados à gestão, como é relatado:

Em relação ao modelo de gestão de uma nova Política de CT\&I/S, a Comissão apontou dois aspectos importantes: o primeiro diz respeito à criação de uma agência de fomento e articulação da pesquisa em saúde externa ao Ministério da Saúde, ainda que situada em sua órbita. Esta proposta vem tendo a adesão de muitos segmentos importantes de atores envolvidos com a pesquisa em saúde. Embora a Comissão reconheça que seu perfil e características mais detalhadas ainda devam ser discutidos, a proposta de sua existência é considerada como um ponto central do modelo de gestão da PCT\&I/S.

O segundo está relacionado à otimização e ao aumento dos recursos financeiros envolvidos no fomento à pesquisa em saúde, condição fundamental para o desenvolvimento da política setorial explicitada na proposta da Commissão. Neste sentido, foram destacadas a recente aprovação do Fundo Setorial de pesquisa em saúde, no âmbito do Fundo Verde-Amarelo e, também, a necessidade de manter viva a proposta original, do próprio Ministério da Saúde, de taxar os lucros da indústria de tabaco e bebidas alcoólicas. Esta idéia vem sendo debatida em vários fóruns, existindo a proposta de estendê-la para outros setores produtivos claramente 'produtores de dívida sanitária'. (destaques no original)

\section{Comissão de EPIDEMIOLOGIA}

Desde que foi instituída, a Comissão de Epidemiologia elaborou quatro planos diretores. O primeiro foi elaborado no seminário Estratégias para o Desenvolvimento da Epidemiologia no Brasil, realizado em Itaparica 
(Bahia), em maio de 1989. A cada cinco anos são realizados seminários com o objetivo de avaliar o que foi implementado, as lacunas e as novas necessidades. Lembramos que essa comissão foi criada em 1984 e que seu fortalecimento, ao longo desses anos, fica evidenciado pela ampla participação no campo da Saúde Coletiva.

Faremos menção mais detalhada ao último plano. No primeiro semestre de 2005, a Comissão de Epidemiologia elaborou o IV Plano Diretor para o Desenvolvimento da Epidemiologia no Brasil, com base em documentos de especialistas da área sobre os três eixos que compõem esse plano - ensino, pesquisa e políticas -, além de programas e serviços de saúde. Ele foi discutido em um seminário no Rio de Janeiro que contou com a participação de expressivos pesquisadores, dirigentes e profissionais do Sistema Único de Saúde (SUS). Estiveram presentes 35 epidemiologistas de 15 programas de pós-graduação das cinco regiões do Brasil e de várias instituições que têm sido as formuladoras das políticas de saúde relacionadas à epidemiologia.

No período de vigência do III Plano Diretor (2000-2004) ocorreram reconhecidos avanços e fortalecimento da epidemiologia nos serviços de saúde, tais como a institucionalização e estruturação da Secretaria de Vigilância em Saúde no Ministério da Saúde (SVS), criação de uma rede de capacitação de recursos humanos para esta área, alguns mecanismos de aperfeiçoamento dos grandes sistemas de informações epidemiológicas, fortalecimento da rede de apoio diagnóstico para a área de saúde pública. Salienta-se em particular a instituição do repasse fundo-a-fundo, mediante critérios epidemiológicos e geográficos, dos recursos do SUS destinados às ações de Vigilância em Saúde trazendo maior estabilidade ao financiamento das ações de Saúde Pública desenvolvidas pelos municípios. Contudo, muitos problemas permanecem inalterados ou mesmo se agravaram neste período, a exemplo da inexistência de política de cargos e salários para os profissionais, o que impede a fixação dos mesmos, principalmente nas áreas mais carentes, com conseqüente descontinuidade das ações nos sistemas locais de saúde e a insuficiência dos recursos do SUS para a Saúde Pública. No que tange ao enfrentamento de situações de saúde inusitadas, a comunidade reconhece que a SVS está adotando algumas iniciativas para a estruturação deste componente da vigilância. No entanto, não tem lançado mão da expressiva capacidade técnica e científica existente no país no campo da epidemiologia. (Teixeira, 2005:231)

Em relação ao ensino, é destacado que, na medida em que se consolidou o SUS e que as atividades de maior interesse para a epidemiologia foram se efetivando, como o Sistema Nacional de Vigilância Epidemiológica e a Vigilância Sanitária, houve necessidade de se repensar a formação de recursos humanos, visando à sua adequação às necessidades 
dos serviços de saúde. Associe-se a isso o fato de que, com a expansão do ensino superior e da pós-graduação e da pesquisa, instaurou-se a necessidade de formação de profissionais mais bem qualificados. Infelizmente, as condições de aproveitamento desses profissionais nem sempre foram favoráveis para as carreiras de docente e de pesquisador.

Dois destaques são dados à formação: orientada para o serviço e para a pesquisa e o ensino. Sem entrarmos em detalhes, citamos como princípios adotados, dentre outros: discutir e difundir novos modelos pedagógicos e de formação, de modo que os modelos tenham uma estrutura modular, hierarquizada e contínua; desenvolver indicadores de avaliação e desempenho; não desvincular a formação e a Saúde Pública/Saúde Coletiva.

Ponto importante no relatório é o dedicado à pesquisa. Após a apresentação de um quadro geral da investigação em saúde no Brasil e em especial da pesquisa epidemiológica, foram discutidos os seguintes tópicos: a produção do conhecimento em epidemiologia, a divulgação científica, a difusão do conhecimento, a ética na pesquisa, teoria e metodologia da pesquisa em epidemiologia. Todos os pontos são apresentados com base na identificação de problemas e, na seqüência, vêm as ações propostas. Citaremos apenas alguns que nos parecem de maior relevância. Em relação à produção, são apresentadas algumas insuficiências, tais como a da articulação de algumas áreas do conhecimento com a política nacional ou com a ciência e tecnologia; incipiência na formação de redes nacionais e internacionais entre os pesquisadores; tendência à especialização do campo. Outros pontos relacionados nesse item referem-se à existência de poucos meios de divulgação; à precariedade da comunicação entre os pesquisadores; à inadequação de alguns dos procedimentos recomendados nos atuais documentos normativos da Comissão Nacional de Ética em Pesquisa (Conep) em relação a estudos epidemiológicos voltados para a Saúde Pública; a questões ainda não resolvidas no campo da incorporação de modelos teóricos na epidemiologia. Para todos os pontos relacionados, foram propostas ações destinadas a aprimorar e avançar o conhecimento e as relações do campo e dos pesquisadores, inclusive no incentivo ao desenvolvimento de metodologias para a avaliação e a incorporação do conhecimento epidemiológico nas políticas públicas, além da análise do seu conseqüente impacto político.

O terceiro ponto tratado no Plano Diretor refere-se à epidemiologia nas políticas, nos programas e serviços de saúde. Sem dúvida, a importância conferida pelos epidemiologistas a esse item expressa-se na criação das Experiências Bem-Sucedidas em Epidemiologia, Prevenção e Controle de Doenças (Expoepi), fórum anual específico para divulgação, discussão e 
premiação de trabalhos e experiências bem-sucedidas no campo da epidemiologia dos serviços de saúde. Revelam os profissionais que há ainda questões a serem resolvidas, como a do "aprimoramento da capacidade dos profissionais em problematizar a sua prática e elaborar perguntas de investigação pertinentes para as políticas de saúde regionais ou locais, e também melhorar a capacidade do uso de métodos de análise epidemiológica" (IV Plano Diretor para o Desenvolvimento da Epidemiologia no Brasil, 2005:29). Ressaltam que continuam válidas as idéias de "manutenção e fortalecimento da articulação entre academia e serviços" (IV Plano Diretor, 2005:30). A simples enumeração dos pontos tratados nesse item revela o destaque concedido à epidemiologia e às questões políticas e de serviços: sistemas de informação; práticas epidemiológicas referentes à análise de situação de saúde; vigilância em saúde; avaliação de programas; recursos humanos e a inserção da epidemiologia nas políticas intra e intersetoriais.

\section{Comissão de Políticas de Saúde, Planejamento e Gestão}

$\mathrm{Na}$ década de 80 , em especial no período de efervescência da Reforma Sanitária, constituiu-se na Abrasco uma Comissão de Políticas de Saúde, Planejamento e Gestão que, posteriormente, se desmobilizaria, vindo a se rearticular no final dos anos 90 . Essa rearticulação incorporou o tema da gestão, que ganhou enorme impulso nessa década, concomitante aos movimentos da reforma do Estado e de suas políticas setoriais, com destaque para a área social. Informações prestadas pela Comissão registram que foi em $1^{\circ}$ de dezembro de 2001, por ocasião da realização do seminário Saúde e Desigualdade - Instituições e Políticas Públicas no Século XXI, que se repensou a questão de se refundar uma Comissão de Políticas de Saúde, Planejamento e Gestão.

Nesta reunião, foi enfatizado o caráter eminentemente acadêmico da iniciativa, visando dentre outros os aspectos referentes à divulgação e intercâmbio das linhas e respectivos focos de pesquisa, a identificação e discussão das questões teóricas e metodológicas relevantes para o desenvolvimento da investigação na Área, a busca de estratégias para o fortalecimento dos grupos de pesquisa em Política, Planejamento e Gestão em Saúde com vistas a melhorar o fluxo de financiamento e a consolidação dos mesmos no âmbito da Saúde Coletiva e o fomento do estreitamento das relações entre os centros acadêmicos e os Gestores do SUS em benefício do aprimoramento do Sistema de Saúde no Brasil. (Informe da Comissão, 2006)

Em comum acordo com a Abrasco, foram definidos os critérios para constituição da Comissão. Dessa forma, estabeleceu-se que deveria 
ter abrangência regional e contemplar instituições mais antigas e mais recentes com produção acadêmica na área. De um rol de 19 instituições inicialmente listadas segundo esses critérios, apenas 12 eram sócias da Abrasco e passaram imediatamente a compor a Comissão: Departamento de Medicina Preventiva e Social/Universidade Estadual de Campinas (DMPS/Unicamp); Departamento de Medicina Preventiva/Faculdade de Medicina da Universidade de São Paulo (DMP/FMUSP); Departamento de Medicina Social/Faculdade de Medicina de Ribeirão Preto/Universidade de São Paulo (DMS/FM Ribeirão Preto/USP); Departamento de Medicina Social/Faculdade de Ciências Médicas da Santa Casa/São Paulo (DMS/FCM Santa Casa/SP); Departamento de Saúde da Coletividade, Faculdade de Medicina do ABC (DSC/FMABC); Escola Nacional de Saúde Pública (ENSP); Faculdade de Saúde Pública/Universidade de São Paulo (FSP/USP); Instituto de Medicina Social/Universidade do Estado do Rio de Janeiro (IMS/Uerj); Instituto de Saúde Coletiva/Universidade Federal da Bahia (ISC/ UFBA); Núcleo de Estudos de Saúde Coletiva/Pernambuco (NESC/PE); Núcleo de Pesquisa Coletiva/Universidade Federal de Minas Gerais (Nescom/UFMG); Núcleo de Estudos de Saúde Coletiva/Universidade Federal do Rio de Janeiro (NESC/UFRJ).

Em 26 de novembro de 2002, foi realizada em São Paulo a primeira reunião da Comissão graças ao apoio da Secretaria de Assistência à Saúde do Ministério da Saúde, contando com oito membros presentes e convidados da Secretaria Municipal de Saúde de São Paulo, da Secretaria de Atenção à Saúde/Ministério da Saúde (SAS/MS) e de integrantes da Equipe de Transição do Governo Federal. A reunião tratou da estruturação interna da Comissão, das propostas da área para o VII Congresso Brasileiro de Saúde Coletiva e da elaboração de uma agenda para a interlocução política com gestores do SUS. As diretrizes para a elaboração de um Plano Diretor da Comissão ficaram para serem efetivadas por meio da realização de uma Oficina de Trabalho Pré-Congresso da Abrasco.

A Comissão foi a responsável pela área temática de Políticas de Saúde, Planejamento, Gestão e Avaliação (Corredor 7) no Congresso de Saúde Coletiva. Nesta atividade, avaliou cerca de novecentos trabalhos, por meio de uma comissão científica composta por 43 pesquisadores, contemplando-se os critérios institucional e regional, mesclando pesquisadores jovens e seniores. A taxa de rejeição dos trabalhos girou em torno de $10 \%$, agrupando-se os aprovados em 18 comunicações coordenadas e 12 painéis, o que traduz a destacada presença da nossa área. A Comissão também propôs nove palestras, abrangendo temas de interesse acadêmico e prático, tais como exclusão, desigualdade, papel 
social do pesquisador, modelos assistenciais, segmentação do sistema de saúde brasileiro e acesso aos medicamentos.

Com o apoio da secretaria executiva do Ministério da Saúde, a Comissão realizou em dois dias uma oficina de trabalho com a participação de cerca de sessenta pessoas pertencentes a instituições de pesquisas de todas as regiões do país, a qual debateu aspectos relativos à inserção da área temática da Comissão no campo da Saúde Coletiva e produziu uma lista de tópicos em conjunto com o gestor do SUS que subsidiou a elaboração do Plano de Trabalho da Comissão.

Destaque-se que a oficina foi um momento de reflexão trazido pela apresentação de palestras e debates que se completaram com a exposição da linha de trabalho do Ministério da Saúde. Conforme o relatório da oficina, os debates apresentaram questões importantes sobre as relações com o Estado e o governo e sobre a necessidade de se articularem as áreas de política, planejamento e gestão tomando a ciência política como centro da atividade do pensar, assim como se constatou a necessidade de promover a articulação entre os diversos centros, a fim de se evitarem centralismos e visões monolíticas nos temas e pesquisas. Dentre as propostas, sobressaiu a intenção de ampliar a visão interinstitucional e de avançar a investigação nessa área.

Retomando as informações da Comissão, destaca-se que ela como desdobramento da Oficina de Trabalho - estabeleceu em 2004 um convênio com o Ministério da Saúde, por meio da secretaria executiva, abrangendo sete projetos: auxílio na constituição e funcionamento da Rede de Apoio à Gestão do SUS; qualificação à gestão descentralizada; avaliação do SUS; regionalização do SUS; levantamento da produção realizada na área de políticas de saúde, planejamento e gestão no Brasil no período de 1999 a 2005; assessoria no processo de acompanhamento e avaliação do Plano Nacional de Saúde; criação de um periódico científico na área de gestão e políticas de saúde. A execução dos projetos envolve todas as instituições componentes da Comissão e se dá de forma sistematizada. Todos se encontram em andamento; inclusive o projeto de uma revista já foi elaborado, estando em negociação com membros do Departamento de Apoio à Descentralização/Ministério da Saúde (DAD/MS).

A Comissão participou ativamente na formulação do VIII Congresso Brasileiro de Saúde Coletiva e do XI Congresso Mundial de Saúde Pública, sendo responsável pelo tema $\mathrm{C}$ do programa. Apresentou 14 eventos (palestras e colóquios) para serem desenvolvidos na ocasião, além de uma Oficina de Trabalho sobre Planejamento e Gestão em Saúde no Brasil. 
No final do documento, a Comissão destaca:

Reafirmamos o nosso compromisso, enquanto Comissão, de desenvolver um trabalho voltado ao conjunto das Instituições que atuam na Área, em benefício do fortalecimento das atividades de ensino, pesquisa e prestação de serviço à comunidade, buscando estreitar as parcerias com os Gestores das três esferas de governo em prol da melhoria do sistema de saúde. (Informe da Comissão de Políticas de Saúde, Planejamento e Gestão, 2006)

\section{Grupos Temáticos}

\section{Acreditação Pedagógica}

A questão da acreditação pedagógica tem a sua história marcada pelas reuniões realizadas no final de 1999,5 quando se definiram as linhas de atuação. Inicialmente, houve necessidade de adesão a um termo até então pouco familiar ao sistema brasileiro, no qual sua utilização vinculase à acreditação hospitalar. Como esclarece Célia Ramos (2003:2),

Este termo encontra-se definido, na literatura de avaliação, como um processo de busca da qualidade a partir de critérios previamente acordados entre partes, em relação a uma prática ou atividade. Não deve ser confundido com labelization ou adequação a alguma ISO. É um mecanismo considerado como o mais adequado para regular a qualidade de cursos voltados explicitamente para serviços.

Essa autora nos informa, ainda, que esse termo tem sido utilizado há mais de 15 anos em vários países, notadamente França, Estados Unidos, Inglaterra e Austrália. Entre nós, o processo foi conduzido pela Escola Nacional de Saúde Pública da Fundação Oswaldo Cruz (ENSP/Fiocruz), mas desde o início a Abrasco e a Coordenação de Aperfeiçoamento de Pessoal de Nível Superior (Capes) constituíram-se como importantes interlocutores, assim como se contou com a assessoria da École National

\footnotetext{
5 O I Seminário de Acreditação Pedagógica foi realizado em 28, 29 e 30 de setembro de 1999, juntamente com o XI Seminário da Coordenação de Cursos Descentralizados, na Escola Nacional de Saúde Pública - Rio de Janeiro. O Relatório Final data de outubro de 1999. O I Seminário Regional de Acreditação Pedagógica foi realizado de 14 a 16 de dezembro de 1999, em Recife (PE); o II Seminário Regional de Acreditação Pedagógica ocorreu em 30 e 31 de março de 2000, em Campo Grande (MS); a Oficina sobre Acreditação dos Cursos Lato Sensu em Saúde Pública, no VI Congresso da Abrasco, em 28 e 29 de agosto de 2000, em Salvador (BA); o III Seminário de Acreditação da Região Sul, em 27 e 28 de julho de 2000, em Porto Alegre (RS); a Oficina de Trabalho do Grupo Político, em 15 e 16 de março de 2001, em São Paulo (SP); a Oficina de Trabalho, em 29 e 30 de maio de 2001, no Rio de Janeiro (RJ).
} 
de Santé Publique de Rennes (França). Não detalharemos as fases das atividades, mas ressaltamos que todo o processo, no período de setembro de 1999 a maio de 2001, foi de construção de consenso em torno da idéia da acreditação, apresentação de um protótipo de instrumento e institucionalização do projeto. De junho de 2001 a agosto de 2003, realizaram-se tarefas que incluíram não somente a finalização do instrumento técnico, mas a de um projeto-piloto, inclusive a participação num fórum internacional realizado na França.

Como informa a nota 5 , muitas foram as atividades desenvolvidas pelo GT. Acrescentamos e salientamos a realizada em 20 e 21 de março de 2003 para o pré-teste do "Manual de Acreditação de Cursos", com a realização de visitas aos três cursos programados.

\section{Comunicaç̃̃o E SaÚde}

Há cerca de seis anos, Pitta e Magajewski (2000) indicavam que no plano acadêmico ocorria um desenvolvimento crescente no campo da comunicação, o mesmo não acontecendo no plano das políticas governamentais. Ressalte-se que o nome genérico - comunicação e saúde envolve a informação, a educação e a comunicação propriamente dita, e nesse documento os autores chamam a atenção para a necessidade de integração dessas três dimensões.

Relato recente desse GT aponta:

Nestes quinze anos, o GTCOM vem buscando articular diferentes campos do conhecimento das Ciências Sociais e Humanas e a Saúde. No entanto, se existem diferentes formas de compreensão e modos de intervir que não são estranhos aos membros do GT, há um consenso mínimo e um conjunto de relações institucionais que aproxima os seus membros, além da própria natureza do objeto de reflexão do grupo que desde a sua criação vem procurando atender aos requisitos e chamamentos de um campo do conhecimento eminentemente transversal ao conhecimento acumulado no campo da Saúde Coletiva, a demandar, portanto, uma prática articulada e cooperativa entre seus membros. (Documento "Memórias de uma Construção", GTCom-Abrasco, 2006:1)

Acentua ainda o documento:

A preocupação com uma reflexão acadêmica entre as relações Comunicação e Saúde data da segunda metade dos anos 80. Pode-se identificar como uma primeira expressão mais estruturada desta preocupação um Encontro promovido pela Fundação Ezequiel Dias/Belo Horizonte, apoiado pela OPAS, em 1989. Desde então são lançadas as bases conceituais para o aprofundamento deste tema. Nesta ocasião, pela primeira vez, alguns dos 
futuros membros do atual GT ABRASCO tiveram o privilégio de encontrar Fernando Lefévre - desde então uma referência na discussão do tema e apoiador incondicional da criação do mesmo. (Documento "Memórias de uma Construção", GTCom-Abrasco, 2006:1)

Ponto importante desse GT tem sido a discussão da constituição de um 'núcleo duro' do seu trabalho - não sem resistências e debates - que ocorreu em diversos momentos: em Belo Horizonte (MG), no Encontro de Ciências Sociais e Saúde, e em seguida no Congresso Brasileiro de Epidemiologia de 1992, no Rio de Janeiro, quando o tema saiu da esfera de um pequeno grupo e foi promovido um primeiro debate ampliado. Nesse cenário, o Grupo Temático Comunicação e Saúde (GTCom) se institucionaliza e inicia a construção de cumplicidades e alianças com outros GTs, em especial de Educação e Informação. Em 1994, no Instituto Brasileiro de Administração Municipal do Rio de Janeiro (Ibam-RJ), deuse o que no GTCom foi denominado de 'Encontro Fundador'. Na ocasião, depois de um longo debate sobre as questões que o GTCom pretendia articular e sobre a necessidade de repensar teorias e metodologias específicas para o campo, foi produzido o Termo de Referência que orientou o debate e as ações do GTCom e de seus membros. O desdobramento desse encontro foi a constituição de cursos de extensão e especialização sobre o tema da comunicação e da saúde.

Os cursos tiveram um papel mobilizador estratégico e se transformaram nos primeiros passos de uma produção científica organizada, de constituição de núcleos institucionais em diferentes regiões do país e de institucionalização e reconhecimento em relação a outros saberes, instituições e campos profissionais. A presença de membros do GTCom orientando em cursos de pós-graduação, no desenvolvimento de projetos de pesquisa, em assessorias e em publicações especializadas demonstra a legitimidade acadêmica dos seus quadros e a capacidade mobilizadora e integradora do tema, que acabou buscando parcerias em grupos de estudos e pesquisas em comunicação de diferentes universidades brasileiras, como a Universidade Federal do Rio de Janeiro (UFRJ), a Universidade Federal de Minas Gerais (UFMG), a Universidade Federal da Bahia (UFBA) e a Universidade de Brasília (UnB). A busca de uma relação mais estreita com o controle público dos serviços e ações de saúde foi objeto de seminário e da publicação, em 1993, do número 1 da série Saúde \& Movimento. Iniciou-se um movimento de parceria com diferentes universidades brasileiras com cursos em comunicação e cultura. Uma conseqüência desse movimento de alianças foi o lançamento, no 'Abrascão' de 1995, em Águas de Lindóia (SP), do livro Saúde e Comunicação: visibilidades e silêncios, 
organizado por Áurea M. da Rocha Pitta (1995). No temário, diferentes perspectivas, autores, instituições, enfoques: novas tecnologias, poder simbólico, democracia, descentralização do processo decisório e das ofertas de serviços, além de usos da mídia, foram alguns dos temas e problemas apresentados para o campo.

Dentre outras atividades do GT, destacam-se as mostras de filmes. O sucesso da iniciativa, ampliando as linguagens e os modos de enunciação da saúde, permite afirmar que ela se desloca do campo do GTCom e, cada vez mais, é parte dos encontros da Abrasco. Promovendo encontros, oficinas, mesas-redondas, painéis e fazendo da comunicação um campo de reflexão, debates acadêmicos e novas práticas, o GTCom insiste em articular a luta política com o debate acadêmico (Documento "Memórias de uma Construção", GTCom Abrasco, 2006:1).

\section{Educação Popular e SaÚde}

Ao relatar a história desse grupo, os participantes retomam o III Simpósio Interamericano de Educação em Saúde, realizado no Rio de Janeiro em 1990, quando se iniciou a articulação nacional dos grupos, profissionais e militantes dos movimentos populares que estavam interessados em educação popular. Em 1991, ocorreu o I Encontro Nacional de Educação Popular em Saúde, em São Paulo, quando foi realmente articulada a organização da área. Nos anos seguintes, realizaram-se encontros, grupos de debates acadêmicos, publicações, mas o grau de participação era pequeno e não havia maior formalidade na organização. Foi com a oficina realizada no Rio de Janeiro, em dezembro de 1998, com o apoio institucional da Escola Nacional de Saúde Pública, que se criou a Rede de Educação Popular em Saúde, ampliando os objetivos de maior integração entre os profissionais latino-americanos e de reorientação das políticas sociais no sentido de torná-las mais participativas. A proposta de criação do grupo temático da Abrasco foi apresentada em agosto de 2000, a fim de institucionalizar as atividades até então desenvolvidas. Somente em 15 de abril de 2005, o GT de Educação Popular e Saúde rediscutiu a sua organização e dinamização, em especial a vinculação da Rede de Educação Popular aos eventos da Abrasco.

Em relação à Rede de Educação Popular, verificamos que a sua ampliação vem ocorrendo, sendo que hoje são 842 membros cadastrados. O boletim Nós da Rede é editado pela Universidade Federal de São Carlos (UFSCar), com tiragem de cinco mil exemplares, impresso e distribuído com o apoio da Secretaria de Gestão do Trabalho e Educação em Saúde/ 
Ministério da Saúde (SGTES/MS). Entre as iniciativas do GT destacam-se a edição dos Cadernos de Educação Popular em Saúde e do Almanaque de Educação Popular em Saúde, em parceria com o Departamento da Gestão da Educação na Saúde/Ministério da Saúde (Deges/MS) e a Articulação Nacional de Movimentos e Práticas de Educação Popular e Saúde (Aneps).

Diante da importância crescente assumida pela questão da educação popular, inclusive com a criação da Secretaria de Gestão do Trabalho e da Educação em Saúde, o GT ampliou o seu papel para além da participação em eventos, desenvolvendo linhas de ação mais consistentes, incluindo a produção de conhecimentos e a interação com outros movimentos sociais. Apresentam-se como objetivos do GT para o período 2005-2007:

- a formação ampliada de recursos humanos em saúde no nível de pós-graduação, especialmente cursos de especialização, cursos de atualização e processo de formação para trabalhadores do SUS;

- a promoção de encontros científicos periódicos para discutir e aprofundar a temática;

- a divulgação das reflexões teórico-metodológicas do campo por meio de publicações como livros, artigos, hipertextos, boletins, listas de discussão e sites.

O GT é formado atualmente por um coordenador, dois vicecoordenadores e comissão executiva constituída por 11 membros. São participantes institucionais, além dos participantes individuais: Rede de Popularização da Ciência e da Tecnologia na América Latina e no Caribe (Rede-POP), Residência em Saúde da Família e Comunidade (Integrada e Médica)/Grupo Hospitalar Conceição-GHC (Rio Grande do Sul), Departamento de Saúde Pública da Faculdade de Medicina/Universidade de Brasília (UnB), Pós-Graduação em Saúde Coletiva/Universidade Comunitária Regional de Chapecó (Unochapecó - Chapecó, Santa Catarina).

\section{GÊNero E SaÚde}

O GT foi criado em 1995, durante o III Congresso Brasileiro de Epidemiologia, realizado em Salvador (BA), e nos primeiros anos de funcionamento contou com o apoio da Fundação Ford e da Organização Pan-Americana da Saúde (Opas). Segundo documento recente,

Ao longo dos seus dez anos de funcionamento, o GT tem buscado fortalecer os vínculos e interlocução entre a academia, os serviços de saúde e os movimentos sociais, particularmente o de mulheres, visando tornar o 
conhecimento acadêmico cada vez mais útil e acessível para os profissionais e demais atores sociais comprometidos com a saúde das mulheres e captar demandas emergentes de produção de conhecimento e formação de profissionais. Também tem procurado contribuir para a incorporação da perspectiva de gênero na compreensão de fenômenos de interesse na área de Saúde Coletiva, abordando novos temas e revisitando antigos. Com isso, pretende criticar o essencialismo das explicações correntes para as diferenças no adoecimento e morte de mulheres e homens e fortalecer abordagens alternativas para os fenômenos, contribuindo para ampliar o tradicional debate sobre as desigualdades sociais em saúde. (Informe do GT Gênero e Saúde, 2006)

A própria temática, bastante diversificada, inclui diferentes profissionais procedentes da epidemiologia e das ciências sociais, tais como: reprodução, juventude, violência doméstica e sexual, Aids e outras doenças sexualmente transmissíveis (DST), controle social, monitoramento e avaliação de políticas e programas para mulheres.

O GT também tem participado de eventos nacionais e internacionais (I Encontro Latino-Americano de Saúde, Eqüidade e Gênero Abrasco/Asociación Latinoamericana de Medicina Social (Alames), 1999; e II International Congress Women Work Health - Fiocruz/Universidade Federal de São Paulo (Unifesp)/Abrasco/Rede Nacional Feminista de Saúde e Direitos Reprodutivos, 1999. Em relação às publicações, editou duas coletâneas (Costa, Merchan-Hamann \& Tajer, 2000; Villela \& Monteiro, 2005).

O grupo temático está representado na Comissão Intersetorial de Saúde da Mulher, uma das comissões assessoras do Conselho Nacional de Saúde, o que possibilita a articulação entre a produção acadêmica e a elaboração de políticas públicas em saúde.

Como forma de comemoração dos seus dez anos, em 2005, durante o IV Congresso de Ciências Sociais e Saúde, o GT realizou uma oficina de avaliação dos avanços e lacunas na incorporação da perspectiva de gênero no âmbito da produção do conhecimento em Saúde Coletiva. A coordenação do grupo temático avaliou:

A oficina apontou que gênero já é assumido como um recorte transversal em um volume significativo de pesquisas na área, do mesmo modo que a idéia de integralidade, conceito que estabelece com gênero uma área de fronteira. (...)

São apontadas algumas lacunas, em especial relacionadas ao campo dos estudos epidemiológicos, quando aparece confundido com a noção de sexo, sendo tomado como variável ou categoria empírica e não como categoria analítica. No campo dos estudos de planejamento e políticas de saúde, a incorporação da perspectiva de gênero ainda é esporádica e muitas vezes incipiente. Ademais, a operacionalização de políticas baseadas na noção de 
gênero não é uma tarefa simples, e muitas vezes essa intenção se traduz apenas na maior oferta de serviços de saúde para mulheres. (Informe do GT Gênero e Saúde, 2006)

Promoção da SAÚde

Durante o VI Congresso de Epidemiologia, em Recife (PE), em 19 e 20 de junho de 2004, o GT de Promoção da Saúde apresentou uma intensa discussão sobre o tema, inclusive com a produção de importantes contribuições para a construção conceitual do campo, para a melhor compreensão das práticas orientadas pela estratégia promocional e para a construção de uma base programática mais consistente e operacional. Por decisão do conjunto dos participantes, ficou estabelecido que o debate não deveria ser fechado em termos de um documento ou relatório final, mas deveria prosseguir buscando ainda novas contribuições de outras organizações e grupos, principalmente extra-setoriais.

Em termos conceituais, reafirmou-se que a Promoção da Saúde (PS) tem como foco a complexidade e o caráter socialmente determinado dos processos saúde-doença, valorizando o enfoque positivo e ampliado de saúde, já presente inclusive na Constituição Brasileira. No plano organizacional, foi considerado que o tema da promoção, por envolver considerações e propostas em todo o campo da Saúde Coletiva, transborda os limites do GT e cruza transversalmente as temáticas de praticamente todos os GTs da Abrasco.

Ficou estabelecido que o GT deve considerar como seu âmbito de atuação o esforço de pesquisa e formação em Promoção da Saúde (PS) em todos os níveis, tanto nas instituições acadêmicas como nas organizações de gestão em saúde, visando expandir os conhecimentos teóricos e práticos no campo - além da tarefa permanente de advocacy pela saúde e pela construção de políticas públicas integradas em prol da qualidade de vida de indivíduos (autonomia) e grupos sociais (eqüidade), conforme o Relatório do GT Promoção da Saúde. ${ }^{6}$

\footnotetext{
${ }^{6}$ Dentre as reuniões de que o GT participou, citamos: Seminário Promoção da Saúde no Contexto do Desenvolvimento Local Integrado e Sustentável, ENSP/Fiocruz, julho de 2002; Pré-III Conferência Regional Latino-Americana de Promoção e Educação em Saúde, Faculdade de Saúde Pública da Universidade de São Paulo (FSP/SP), 10/11/2002 (junto com o DLIS); Fórum Social Mundial, Porto Alegre (RS), 23/1/2003; Oficina no VII Congresso da Abrasco, Brasília, 29/7 a 2/8/2003; Oficina de Trabalho no VI Congresso Brasileiro de Epidemiologia, Recife (PE), 19 a $23 / 6 / 2004$.
} 


\section{SaÚde do Trabalhador}

Cita-se como marco inicial para a construção do GT a Reunião Nacional sobre Ensino e Pesquisa em Saúde Ocupacional, realizada em Campos do Jordão (SP), em 1983. Mesmo com a realização da I Conferência Nacional de Saúde do Trabalhador, em 1986, que deflagrou importantes questões sobre as relações trabalho e saúde, foi somente em 1994, durante a realização do V Congresso Paulista de Saúde Pública, em Águas de Lindóia (SP), que o GT se constituiu. Para os integrantes da área,

Os dez anos seguintes se caracterizaram por uma permanente contradição entre a alta relevância da temática e a carência de espaços consolidados no interior do setor saúde. Reflexo dessa situação é a existência de experiências pontuais nos serviços de saúde e a limitada estruturação em instâncias acadêmicas. (Informe do GT Saúde do Trabalhador, 2006)

\section{Prossegue o Informe:}

Nesse período, o GT tem realizado muitas atividades e um destaque importante tem sido as questões relativas aos acidentes de trabalho, com a revisão das informações oficiais de acidentes de trabalho e a formulação de propostas multicêntricas de aprofundamento, mas não receberam o apoio necessário das agências de fomento, até o presente. (Informe do GT Saúde do Trabalhador, 2006)

\section{Ainda nesse Informe é salientado que}

Uma das tentativas do grupo é a de trabalhar em rede, disseminando informação sobre o GT via internet com o intuito de ampliar o número de participantes e o envolvimento em atividades diversas. Destaca-se, ainda, a participação do GT na organização da $3^{\text {a }}$ Conferência Nacional de Saúde do Trabalhador, realizada em 2005, decisiva na melhoria dos debates, principalmente pela colaboração prestada por alguns dos membros na elaboração de textos de apoio e na edição de um número especial da revista Ciência \& Saúde Coletiva. (Informe do GT Saúde do Trabalhador, 2006)

Como proposta, o GT apresenta o grande desafio: a realização de um primeiro Congresso Nacional de Saúde do Trabalhador em 2007, com vistas a aprofundar o campo da saúde do trabalhador. Pretende-se, também, proceder a articulações com os países latino-americanos, inclusive discutindo a possibilidade de se organizar um congresso de âmbito latinoamericano.

Num sentido crítico, mas altamente revelador da maturidade do campo, o GT assinala:

Em síntese, numa análise crítica desses 20 anos, constatamos que estivemos muito voltados para nós mesmos, dadas as deficiênicias da estruturação da área nos serviços de saúde e nas universidades. As redes propostas sempre 
tiveram uma característica endógena, o desafio atual é, além de aprofundarmos conceitual e praticamente a natureza da nossa perspectiva acadêmica, costurar relações amplas com parceiros fora do setor da saúde estrito senso. (Informe do GT Saúde do Trabalhador, 2006)

\section{SAÚde e Ambiente}

O Grupo de Trabalho Saúde e Ambiente foi organizado em 2001 e desde o início contou com o apoio da Coordenação Geral de Vigilância Ambiental/Fundação Nacional de Saúde (CGVAM/Funasa), atual Serviço de Vigilância Sanitária, e da Representação da Organização Pan-Americana da Saúde/Organização Mundial da Saúde (Opas/OMS) no Brasil. A primeira oficina foi realizada nos dias 21 e 22 de maio de 2001, na sede da Opas/ OMS em Brasília. Naquele momento, o GT procurou pautar suas atividades a fim de construir sua identidade, congregando profissionais e sendo contemporâneo das questões que relacionam saúde e ambiente. Essa fundamentação tornou-se um dos princípios do grupo, seja no campo do conhecimento, seja no desempenho de seu papel político na proposição de estratégias de ação. Assim, numa perspectiva de integração e articulação entre os diversos órgãos que tratam do assunto, a agenda deveria contemplar três questões básicas: a poluição, a água e a floresta.

Além dessa pauta ampla, outros temas deveriam ser abordados: as questões urbanas (áreas metropolitanas), as questões rurais (uso do solo, agrotóxicos e transgênicos), controle químico do setor saúde. A longa pauta desenvolvida incluiu questões teórico-conceituais e metodológicas para tratar a interface saúde/ambiente, as relações com as políticas, os programas e os serviços de saúde, a vigilância em saúde e ambiente, além da pesquisa em saúde e ambiente, conforme a Versão Preliminar do Plano Diretor Saúde e Ambiente, elaborada em 2003.

Em 2003, o Relatório do GT assinalava que o grupo estava constituído por vinte membros procedentes de diversos estados e instituições, incluindo alguns técnicos do Ministério da Saúde e da Opas.

O GT teve participação de destaque no VII Congresso da Abrasco, organizando um corredor temático com uma mesa de debates, sete painéis, duas conferências e três palestras. Saliente-se, ainda, que os membros do GT produziram oito artigos para a Revista Brasileira de Epidemiologia e colaboraram com diversos eventos nacionais: Agenda 12 Brasileira, elaboração da publicação Geo-Brasil, Seminário na Câmara dos Deputados sobre Legislação do Saneamento, instituição do Prêmio Milton Santos em colaboração com a Fiocruz, edição de um livro sobre ambiente com a Fiocruz, Dia Mundial da Saúde (tema Saúde Ambiental Infantil), I Seminário 
Nacional de Saúde e Ambiente com Controle Social, participação na Sociedade Brasileira para o Progresso da Ciência (SBPC). Lembramos que, durante o VII Congresso da Abrasco, o GT reuniu cinqüenta convidados em uma oficina que foi altamente importante na fixação dos encaminhamentos do grupo, conforme o Relatório do GT Saúde e Ambiente, 2003.

\section{SaÚde dos Povos Indígenas}

O GT Saúde dos Povos Indígenas foi criado em 2000, como resultado da oficina de trabalho sob os auspícios do Centro de Pesquisas Leônidas e Maria Deane, da Fundação Oswaldo Cruz, em Manaus (AM), da qual participaram pesquisadores vinculados a instituições de ensino e pesquisa em saúde das diversas regiões do país. O grupo que tomou parte dessa oficina foi constituído com base em indicações da Abrasco e da Associação Brasileira de Antropologia (ABA), cada qual com quatro representantes.

As origens do interesse sobre essa temática prendem-se à

intensificação dos debates acerca da importância da categoria etnia e, em particular, dos indígenas, como relevante no debate acerca das iniqüidades sociais e em saúde no Brasil. Estudos recentes têm demonstrado amplamente o quadro de marginalização sócio-econômica [sic] e política no qual estão inseridas as sociedades indígenas no Brasil, com graves impactos sobre sua saúde. (Documento GT Saúde dos Povos Indígenas, jun.2006)

Devem ser consideradas ainda as peculiares características da morbimortalidade indígena que mostram proeminência das doenças infectoparasitárias, mas que apresentam, também, um quadro no qual se observa:

a rápida emergência das doenças crônicas não transmissíveis, em especial a obesidade, diabetes mellitus e hipertensão. As conseqüências dessa 'sobreposição' de perfis epidemiológicos (para os indivíduos, as comunidades e os serviços de saúde) são amplas e de difícil caracterização devido à precariedade das fontes de informação sobre a saúde das populações indígenas. A tendência à urbanização de parcela expressiva desse contingente populacional torna ainda mais complexa a análise do processo saúde-doença indígena, pois pouco se conhece acerca de suas condições de vida nas cidades. (Documento GT Saúde dos Povos Indígenas, jun.2006)

Concorreu também para o crescente interesse nessa temática a promulgação, em 1999, da Política Nacional de Atenção aos Povos Indígenas, com a proposta de 'distritalização' da atenção à saúde dessas populações, por meio da implantação de 34 Distritos Sanitários Especiais Indígenas (DSEI) em todo o país. Verificou-se ainda, em muitos distritos, a 
terceirização da gestão e dos serviços, o que se deu mediante convênios firmados entre o nível federal - representado pela Fundação Nacional de Saúde (Funasa) - e diversas organizações não-governamentais, missões religiosas e, mais recentemente, fundações universitárias.

Os autores do documento que estamos utilizando indicam:

Durante os seus cinco anos de funcionamento (2000-2005), o GT tem atuado em várias frentes. No campo político, uma das conquistas mais importantes foi a obtenção de uma vaga de representação junto à CISI (Comissão Intersetorial de Saúde Indígena), vinculada ao Conselho Nacional de Saúde. Trata-se de espaço estratégico no qual são discutidos temas correntes relacionados à política de atenção à saúde indígena no país. Outro papel importante desempenhado pelo GT foi marcado por sua participação junto ao DECIT/MS, que resultou na elaboração das 'prioridades de pesquisa em saúde indígena', contempladas em vários editais recentes do DECIT-CNPq. (destaques no original)

Destacam os autores a importância dos congressos anuais promovidos pela Abrasco nas áreas de Saúde Coletiva, epidemiologia e ciências sociais, os quais têm servido como espaço fundamental de atuação do GT. Em 2002, durante o V Congresso Brasileiro de Epidemiologia, foram inauguradas as oficinas de trabalho de 'saúde indígena'. Desde então, realizaram-se quatro oficinas, dedicadas aos seguintes temas: "Saúde e Epidemiologia das Populações Indígenas no Brasil” (Curitiba, 2002), "Políticas Públicas e Saúde das Populações Indígenas" (Brasília, 2003), "Indicadores Epidemiológicos, Avaliação de Serviços e Saúde Indígena" (Recife, 2004) e "A Antropologia e os Desafios da Saúde Indígena no Brasil" (Florianópolis, 2005). Cerca de 280 pesquisadores, estudantes e técnicos oriundos de todos os estados e, freqüentemente, representantes dos mais distantes Distritos Sanitários Especiais Indígenas (DSEI) participaram dessas oficinas. Vale mencionar que as oficinas de saúde indígena promovidas por esse GT tornaram-se fóruns únicos no qual pesquisadores e profissionais dos serviços de saúde debatem temas de interesse comum e discutem os resultados de pesquisas recentes na área. Os participantes das oficinas buscam ainda identificar lacunas do conhecimento, propor linhas de investigação e formas de articulação das instituições de pesquisa e ensino com os serviços de saúde.

Um importante produto do GT nesse primeiro período de atividade foi a publicação da coletânea Epidemiologia e Saúde dos Povos Indígenas no Brasil (Coimbra Jr., Santos \& Escobar, 2003), com apoio da Fundação Ford, lançada em Brasília durante o VII Congresso Brasileiro de Saúde Coletiva. É constituída de uma seleção de textos e experiências que foram discutidas durante a oficina de trabalho realizada em Curitiba (PR), em 
2002. Anteriormente, em 2001, fora lançado um fascículo temático de Cadernos de Saúde Pública (volume 17, número 2), intitulado Saúde dos Povos Indigenas no Brasil: perspectivas atuais, que congregou reflexões e estudos de caso sobre temas diversos e reuniu vários participantes do GT.

Destaque-se na atualidade o trabalho do GT com o objetivo de consolidar articulações com agências governamentais - Fundação Nacional do Índio (Funai) e Funasa - e estabelecer parcerias com outras associações científicas, particularmente com a Associação Brasileira de Antropologia (ABA) e com a Associação Brasileira de Estudos Populacionais (Abep), por meio de seu GT de Demografia Indígena, inclusive realizando oficinas de trabalho conjuntas durante os congressos anuais da Abep. Como primeiro produto dessa parceria, foi publicada importante coletânea que congregou as pesquisas mais recentes sobre o tema (Pagliaro, Azevedo \& Santos, 2005). Vale mencionar que se trata do primeiro livro publicado no país sobre demografia indígena. Outro importante produto da colaboração entre os GTs Saúde Indígena da Abrasco e Demografia Indígena da Abep consiste na primeira análise sistemática do componente indígena dos censos de 1991 e 2000, que se tornou possível graças ao apoio do Instituto Brasileiro de Geografia e Estatística (IBGE). Esse estudo resultou na publicação de um livro que integra a série "Tendências Demográficas", publicada pelo IBGE, e intitula-se Uma Análise dos Indígenas com Base nos Resultados dos Censos Demográficos 1991 e 2000 (IBGE, 2005).

No final do documento, os autores reconhecem como desafios: a implementação e consolidação da nova política de atenção à saúde indígena - envolvendo centenas de milhares de usuários, além de agências governamentais e não-governamentais -, sem perder de vista a imensa sociodiversidade indígena, bem como a heterogeneidade das demandas e de perfis epidemiológicos verificados entre os vários DSEI; o papel estratégico a ser desempenhado pelas pesquisas em Saúde Coletiva, sempre que integradas às atividades de ensino e formação de recursos humanos nas diversas instâncias do sistema formador (graduação e pós-graduação); e a incorporação dos conhecimentos gerados às ações de saúde. $O$ documento enfatiza a necessidade do fortalecimento de parcerias interregionais que agreguem serviço-ensino-pesquisa e a recuperação da identidade cultural indígena.

\section{INFORMAÇÃO EM SAÚDE}

No relato do GT, o ponto inicial refere-se à proposta desse grupo temático no sentido de "Constituir-se em um espaço aberto e plural de 
debate, construção e sistematização de propostas relacionadas a um Projeto Nacional para o campo da Informação em Saúde comprometido com a melhoria da Saúde da população brasileira". A idéia de sua constituição surgiu como um dos produtos da Oficina de Trabalho realizada no II Congresso Brasileiro de Epidemiologia, em Belo Horizonte (MG), em 1992, e tomou como referência os seguintes pontos:

1.O diagnóstico em torno da situação das informações em saúde, evidenciando sua fragmentação, a falta de processos abertos de padronização, a ausência de uma 'cultura do uso da informação' no processo decisório, a fragilidade das estratégias de disseminação e da elaboração de preceitos éticos e de segurança que protejam a privacidade do cidadão.

2.Coerência com a tendência internacional, apesar do atraso, de modo a se tornar um incremento na produção de conhecimento relacionado à informação em saúde, suscitando novas demandas para as instituições de pesquisa e ensino no Brasil.

3. A constatação da necessidade de novos conteúdos nos processos de capacitação que propiciem novas habilidades e competências aos profissionais responsáveis pela gestão da informação, impondo que sejam aprofundadas as reflexões sobre qual o perfil desse profissional em face do acelerado processo de inovações tecnológicas nesse campo.

O GT adota um conceito amplo de informação em saúde, englobando as informações sociais e demográficas e incorporando, entre seus membros, representantes da Associação Brasileira de Estudos Populacionais (Abep). Com o referencial da inter e da transdisciplinaridade, a primeira composição do GT Informação em Saúde contou com participantes das seguintes instituições: Universidade de São Paulo (USP), Universidade Federal da Bahia (UFBA), Fiocruz, Departamento de Informação e Informática do Sistema Único de Saúde (Datasus) e, pela Abep, IBGE, Fundação Sistema Estadual de Análise de Dados Estatísticos (FSEADE) e Universidade do Estado do Rio de Janeiro (Uerj).

O Informe do GT Informação em Saúde (2006) relata que

Tensões marcaram a constituição desse Grupo Temático: a interlocução com outras comissões e GT(s) da ABRASCO, principalmente com a Comissão de Epidemiologia e posteriormente com o GT Comunicação e Saúde. No Brasil, o campo da Informação em Saúde não tem suas delimitações epistemológicas suficientemente legitimadas pelos pares no interior da Saúde Coletiva. Basicamente em função desse diálogo entre campos não estar devidamente aprofundado, a ABRASCO optou pela denominação de 'grupo técnico/ 
temático', por entender que a Informação em Saúde não se constituía em um campo disciplinar que justificasse a formação de uma 'Comissão'. Esse debate no âmbito da Saúde Coletiva permanece atual. (destaques no original)

No início de sua atuação, o GT priorizou: 1) a compatibilização das bases de dados, com a melhoria de sua qualidade; 2) a definição de estratégias de disseminação das informações; 3) a necessidade de um amplo processo de educação permanente dos profissionais responsáveis pela gestão da informação; e 4) elaboração de preceitos éticos sobre o tratamento e uso da informação em saúde que identifica o cidadão. Estes temas continuaram a fazer parte do trabalho do GT, sendo acrescidos e atualizados, destacando-se a elaboração de preceitos éticos sobre o tratamento e o uso da informação em saúde que identifica o cidadão. O GT realizou oficinas de trabalho em todos os congressos de Saúde Coletiva e de epidemiologia, e as contribuições dessas oficinas pautaram discussões posteriores, tanto na academia como nos órgãos gestores do SUS. Como contribuição, redigiu o documento "Informação em Saúde a Serviço da Sociedade", elaborado pelo Ministério da Saúde em 1993, no qual apresenta alguns dos marcos referenciais que orientam até hoje os debates em torno da informação em saúde.

Destaque-se a intensa produção científica e técnica que o GT vem realizando:

ABRASCO/ABEP. Grupo Técnico de Informações em Saúde e População (GTISP). Informação em Saúde a Serviço da Sociedade. In: MINISTÉRIO DA SAÚDE. Uso e Disseminação de Informação em Saúde: subsídios para a elaboração de uma política de informações para o SUS. Brasília: Ministério da Saúde; Abrasco/Oficina de Trabalho - Relatório Final, anexo 01, 1994. p.27-44.

ABRASCO. Informações em Saúde no Brasil: um desafio para a Ciência e Tecnologia. Anais da I Conferência Nacional de Ciência e Tecnologia em Saúde. Brasília: Ministério da Saúde, 1994.

ABRASCO. Oficina de Trabalho Compatibilização de Bases de Dados Nacionais. Informe Epidemiológico do SUS, 6(3):25-33. Brasília: Cenepi/ FNS/Ministério da Saúde, 1997.

MORAES, I. H. S. de \& SANTOS, S. R. R. F. Informação em saúde: os desafios continuam. Ciência \& Saúde Coletiva, 3(1):37-51. Rio de Janeiro: Abrasco, 1998.

MORAES, I. H. S. de \& SANTOS, S. R. R. F. Informações para a gestão do SUS: necessidades e perspectivas. Informe Epidemiológico do SUS, 10(1):4956. Brasília: Ministério da Saúde/FNS/Cenepi, 2001. 
Ao lado dessa produção, outros trabalhos foram realizados de forma colaborativa:

IBGE. Informações para uma sociedade democrática: por uma Política Nacional de Produção e Disseminação de Informações Sociais, Demográficas, Econômicas e Territoriais. Anais da Conferência Nacional de Estatística e da Conferência Nacional de Geografia. Rio de Janeiro: IBGE, 1994.

MINISTÉRIO DA SAÚDE/DATASUS. Diretório de Bases de Dados de Interesse para a Área de Saúde. Brasília: Ministério da Saúde, 1993.

MINISTÉRIO DA SAÚDE. Uso e Disseminação de Informação em Saúde: subsídios para a elaboração de uma política de informações para o SUS. Brasília: Ministério da Saúde; Abrasco/Oficina de Trabalho - Relatório Final, 1994 (o GTISP foi o responsável pela relatoria).

MINISTÉRIO DA SAÚDE/DATASUS. CD-ROM para Disseminação do Sistema de Informações Hospitalares do SUS - SIH/SUS. Brasília, 1995.

Verifica-se que o GT, desde a sua criação, procurou integrar-se aos órgãos públicos e às associações científicas no sentido de lhes dar não somente um caráter científico, mas de atuação direta nos problemas relacionados à informação, a fim de garantir um contínuo processo de democratização e qualificação da informação em saúde.

\section{Considerações Finais}

O presente relato das comissões e dos grupos temáticos não aborda integralmente todas as atividades desenvolvidas, especialmente porque faltam informações sobre alguns grupos temáticos. Embora exista essa falha, percebemos que houve um grande avanço em relação a essas organizações dentro da Abrasco. Formados por especialistas, os GTs têm procurado ultrapassar as fronteiras disciplinares, estabelecendo uma profícua interação entre diferentes profissionais e campos de saberes. As comissões e os GTs também têm desempenhado papel fundamental na organização dos congressos e eventos na área de Saúde Coletiva e mantido estreitas relações com as instituições públicas quando emergem problemas relacionados a suas especificidades temáticas.

\section{Agradecimentos}

Agradecemos às pessoas que nos enviaram as informações, mas esclarecemos que, pelas dimensões deste trabalho, fomos obrigados a fazer recortes e sínteses dos documentos. 


\section{REFERÊNCIAS BIBLIOGRÁFICAS}

BELISÁRIO, S. A. Associativismo em Saúde Coletiva: um estudo da Associação Brasileira de Pós-Graduação em Saúde Coletiva - Abrasco, 2002. Tese de Doutorado, Campinas: Faculdade de Ciências Médicas/Universidade Estadual de Campinas (Unicamp). (Mimeo.)

CANESQUi, A. M. (Org.) Dilemas e Desafios das Ciências Sociais na Saúde Coletiva. São Paulo: Hucitec; Rio de Janeiro: Abrasco, 1995.

COIMBRA JR., C. E. A.; SANTOS, R. V. \& ESCOBAR, A. L. (Orgs.) Epidemiologia e Saúde dos Povos Indígenas no Brasil. Rio de Janeiro: Editora Fiocruz/Abrasco, 2003.

COSTA, A. M.; MERCHAN-HAMANN, E. \& TAJER, D. (Orgs.) Saúde, Eqüidade e Gênero: um desafio para as políticas públicas. Brasília: Editora da Universidade de Brasília, 2000.

GOLDENBERG, P.; MARSIGLIA, R. M. G. \& GOMES, M. H. de A. O Clássico e o Novo: tendências, objetos e abordagens em ciências sociais e saúde. Rio de Janeiro: Editora Fiocruz, 2003.

IBGE. Uma Análise dos Indígenas com Base nos Resultados dos Censos Demográficos 1991 e 2000. Rio de Janeiro: IBGE, 2005. (Série Tendências Demográficas)

KAUfMANN, A. El Poder de Las Organizaciones: comportamiento, estructura y entorno. Madri: Ediciones de la Universidad Alcalá de Henares, 1993.

PAgliaro, H.; AZEVEDO, M. \& SANTOS, R. V. (Orgs.) Demografia dos Povos Indigenas no Brasil. Rio de Janeiro: Editora Fiocruz; São Paulo: Abep, 2005.

PITTA, A. da R. (Org.) Saúde \& Comunicação: visibilidades e silêncios. São Paulo: Hucitec/Abrasco, 1995.

PITTA, A. M. da R. \& MAGAJEWSKI, F. R. L. Políticas nacionais de comunicação em tempos de convergência tecnológica: uma aproximação ao caso da saúde. Interface - Comunicação, Saúde, Educação, 4(7):61-70, 2000.

RAMOS, C. L. Acreditação em Cursos de Saúde Pública: visando a qualidade no ensino lato sensu. Fev.2003. (Mimeo.)

TEIXEIRA, M. G. IV Plano Diretor para o Desenvolvimento da Epidemiologia no Brasil. Revista Brasileira de Epidemiologia, 8(3):231-233, set.2005.

VILlelA, W. \& MONTEIRO, S. (Orgs.) Gênero e Saúde: Programa de Saúde da Família em questão. Rio de Janeiro: Abrasco/United Nations Population Fund (UNFPA), 2005. 

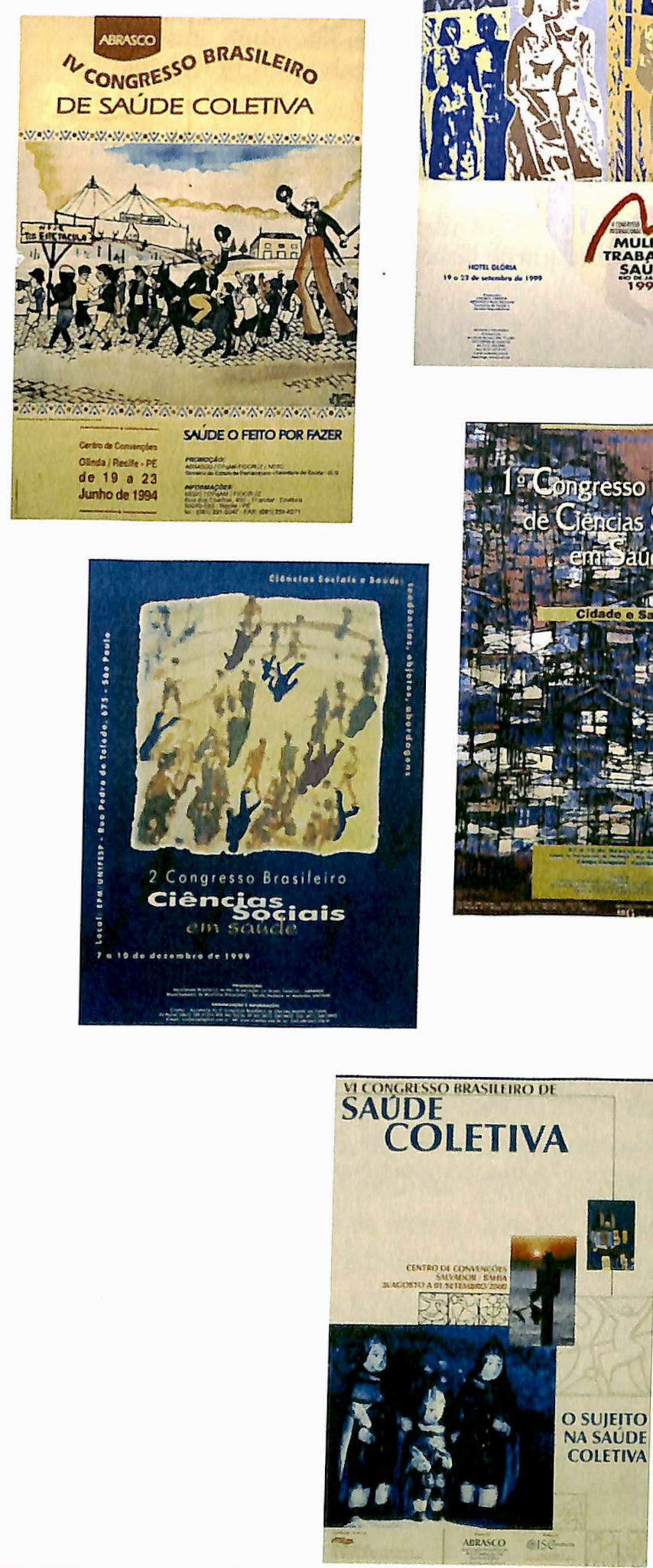
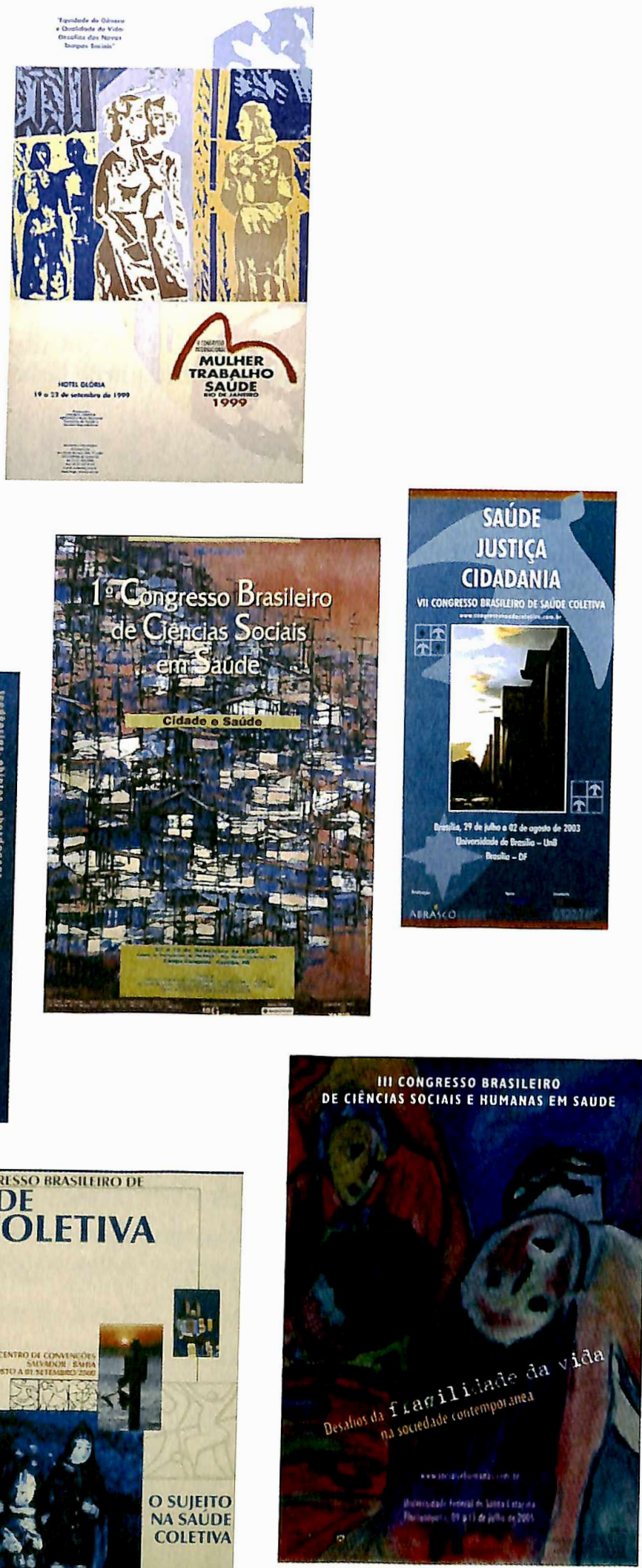